\title{
MujERES HACKER, SABER-HACER Y CÓDIGO ABIERTO: TEJIENDO EL SUEÑO HACKFEMINISTA
}

\section{Hacker Women, Know-How, And Open SOURCE: WeAVING THE HackFeminist Dream}

\author{
Irene Soria-Guzmán* \\ DOI: http://dx.doi.org/10.29043/liminar.v19il.806
}

Resumen: Este artículo da cuenta, de manera descriptiva, de algunas prácticas y saberes de las mujeres hacker en relación con el dominio y la apropiación de técnicas computacionales especializadas, a partir de una metodología feminista que supone la exploración situada de quien realiza esta investigación. Estos hallazgos, aunque aún en proceso de análisis, pretenden esbozar una posible apropiación tecnológica que aporte al movimiento feminista del siglo XXI, a través de los principios de la cultura hacker, para tejer una nueva y posible lucha hackfeminista.

Palabras clave: hackfeminismo, cultura hacker, tecnofeminismo, mujeres y tecnología, hacktivismo, software libre, apropiación tecnológica.

\begin{abstract}
This article gives a descriptive account of some of the practices and knowledge of women hackers in relation to the mastery and appropriation of specialized computational techniques, based on a feminist methodology that involves the situated exploration of the person conducting this research. These findings, although still in the process of analysis, aim to outline a possible technological appropriation that will contribute to the feminist movement of the 2lst century, through the principles of hacker culture, to build a new and viable feminist hacker struggle.
\end{abstract}

Keywords: hacker feminism, hacker culture, techno-feminism, women and technology, hacktivism, free software, technological appropriation.

\footnotetext{
* Irene Soria Guzmán. Estudiante en el Doctorado en Estudios Feministas en la Universidad Autónoma Metropolitana, Unidad Xochimilco, México. Profesora-investigadora en la Universidad del Claustro de Sor Juana y la Universidad Latinoamericana, México. Activista de la cultura libre, representante líder de Creative Commons México. Temas de especialización: tecnología y mujeres, software libre, cultura hacker, ciberfeminismo, tecnofeminismo, hackfeminismo, comunicación y medios digitales, tecnología crítica. Correo electrónico: idem24@gmail.com. ORCID: https://orcid.org/0000-0002-1719-1734
}

Enviado a dictamen: 13 de abril de 2020. Aprobación: 14 de septiembre de 2020. 


\section{Introducción}

S ituar el cruce entre mujeres y tecnología dentro de la estructura neoliberal y capitalista en la era digital actual, caracterizada por la vigilancia masiva, el big data, la violencia en línea, la hiper mediación y el control por parte de las empresas proveedoras de servicios y fabricantes de software, así como yuxtaponerla con el planteamiento político del feminismo, supone la consideración de múltiples factores, como, por ejemplo: el rastreo histórico de la relación de las mujeres con la técnica: la problematización de la generización de la tecnología; el papel de las mujeres en la tecnociencia; ${ }^{1}$ la reflexión y la postura crítica sobre los conocimientos técnicos del cómputo, asociados -y, en gran medida, forjados - desde una visión hegemónica y androcéntrica; la escasa participación de mujeres diversas en el diseño y desarrollo de las tecnologías de la información y la comunicación (TIC), y las implicaciones que esto podría tener para la construcción de un futuro que se vislumbra dominado por tecnologías computacionales; no debe olvidarse, por supuesto, el contexto local de los países del sur global, que se encuentran en total desventaja en el marco de esta política neoliberal, que avanza en el extractivismo de datos personales como materia prima para su funcionamiento.

La negación, el alejamiento y la poca injerencia que hemos tenido las mujeres en el desarrollo y diseño de ciertas tecnologías computacionales, más allá del uso y consumo de aparatos y dispositivos móviles, ha sido uno de los temas en los debates feministas de las últimas décadas. Además de ello, existe una severa problemática pocas veces abordada desde las ciencias sociales, vinculada al cierre de código fuente ${ }^{2}$ de programación, que ocurrió a mediados de la década de los setenta e impide un conocimiento profundo y la apropiación del software que hace funcionar los equipos de cómputo y la mayor parte de los servicios digitales que usamos hoy en día. Esto se encuentra estrechamente relacionado con la privatización de la tecnología y de los saberes específicos por parte de los grandes corporativos tecnológicos, también conocidos como GAFAM. ${ }^{3}$
Frente a la nueva forma de privatización del conocimiento en tanto cierre del código fuente del software, aparecieron algunos movimientos de resistencia como el del software libre en la década de los ochenta en Estados Unidos, vinculado con la cultura hacker de la que se hablaba desde los sesenta, y que plantean una oposición al control tecnológico por medio de la apertura del código fuente y el libre uso de los programas de cómputo. Las mujeres hacker de este movimiento, aunque presentes, siguen siendo pocas, y falta saber mucho al respecto de sus motivaciones, experiencias, subjetividades y estrategias para insertarse en un campo de saber masculinizado.

Es por ello que en el proyecto de investigación del cual parte este artículo busco identificar las prácticas, saberes y experiencias de las mujeres hacker en tanto su condición de género, y reconocer en ellas una construcción de sus "saber-hacer", ${ }^{4}$ en la que quizá se vislumbre una fisura en el sistema de control tecnológico hegemónico. Probablemente dichos "saber-hacer" representen una forma de ejercicio de poder de estas mujeres, y con ello una posibilidad de apropiación tecnológica que aporte al movimiento feminista del siglo XXI, a través de los principios de la cultura hacker, para dar paso con ello a una nueva y posible lucha hackfeminista. Sin embargo, cabe aclarar que los alcances de este artículo se limitan a los primeros hallazgos y a esbozar el devenir hacker, en tanto inquietudes, reflexiones y cómo adquieren este conocimiento especializado ocho mujeres hacker a las que entrevisté hasta el momento de la publicación de este artículo.

\section{Técnica, mujeres y código de programación}

El debate teórico de la tecnología dentro del feminismo se ha venido dando desde la década de los setenta, con la discusión en torno a las tecnologías domésticas y su relación con el dominio capitalista y patriarcal. La vinculación de las identidades sexo-genéricas — generalmente binarias - con actividades públicas y privadas que a su vez parten de la división sexual del trabajo, se traslada a las tareas que se realizan dentro del hogar, las cuales se asocian con las mujeres, y las que se realizan fuera del hogar, asociadas a los varones. Igualmente, 
dichas actividades se relacionan con el uso de ciertos objetos tecnológicos (Natansohn, 2013).

Asimismo, gracias a la genealogía de algunas ciberfeministas (Cruells, Vergés y Haché, 2014), sabemos que, si bien han sido pocas las mujeres que han participado activamente en las tecnociencias, las que lo han hecho han sido invisibilizadas o poco estudiadas en comparación con sus contrapartes masculinos. De igual forma, la mirada del feminismo interseccional ha situado en el debate el intrincado cruce de las categorías de raza, clase y país de origen como parte del engranaje que configura la brecha digital de género.

Sin embargo, entender la tecnología como el discurso de la técnica (techné), es decir, como "el dominio restringido y específico de los útiles, los instrumentos, cuando no sólo de las máquinas", implica también seguir la misma definición de Bernard Stiegler (2002:145) sobre la técnica en tanto "saber" que transforma materiales, es decir, el conjunto de saberes que "producen" o que "hacen"; entonces, podríamos hablar de la técnica como un "saber-hacer", y llevar la problemática un poco más atrás para remitirnos al despojo ancestral de particulares "saberes" del que han sido objeto las mujeres.

La antropóloga Paola Tabet menciona que el papel de las mujeres en el uso de ciertos instrumentos y en su dominio de la técnica está vinculado estrechamente con la división sexual del trabajo, lo que quiere decir que, como consecuencia de su papel de cuidadoras y recolectoras, y al estar relacionadas con la esfera de lo "privado", les es negada la capacidad de extenderse más allá de sus propias fuerzas físicas, de la capacidad de sus manos y de la prolongación de sus brazos en instrumentos complejos que acrecentaran su poder sobre la naturaleza, contrario a lo que pasaba con los varones y el uso de instrumentos para la caza (Tabet, 2005:67). Valdría la pena cuestionarse si este no es un antecedente que precede a la brecha entre mujeres y tecnología que se observa hoy en día, ya que "existe una clara diferenciación con respecto a quiénes hacen determinadas actividades, con qué lo hacen y cómo lo hacen" (Tabet, 2005:119).

Aunado a esto, la cacería de brujas en Europa, que alcanzó su punto máximo entre 1580 y 1630, y que, según
Federici, "resultó ser un elemento esencial para la acumulación primitiva y para la transición al capitalismo" (2004:178), se concentró en la persecución de mujeres que de una u otra manera desafiaron a la estructura de poder de la época: las curanderas de los pueblos, mujeres herederas de un saber empírico relacionado con remedios curativos, herbolaria y conocimientos ancestrales, que para fines reflexivos de esta investigación son reconocidas como mujeres que poseían un profundo dominio de la técnica y que, a su vez, desafiaban a la medicina científica que representaba la muralla del "conocimiento científico indisputable, inasequible y extraño para las clases bajas” (Federici, 2004:278).

De este modo, un eje importante a considerar es el hecho de que las mujeres han sido poseedoras de saberes vinculados con la esfera de lo privado, los cuales han sido denostados y desvalorizados, pues no se han considerado como técnicas complejas que "producen" valor dentro de la lógica capitalista. Los saberes de las mujeres han sido, por lo tanto, subalternos.

Asimismo, la esfera de lo público, asociada a una identidad masculina, se vincula con la ciencia, la técnica, la producción de ideas, la razón, el entendimiento y la maquinaria especializada, mientras que la esfera de lo privado, asociada a las mujeres, se vincula con la naturaleza, la pasión, los sentimientos, los cuidados y la improductividad-no trabajo (Boix y De Miguel, 2002). Quizá sea por eso que a las mujeres se nos relaciona con el poco o nulo manejo de máquinas complejas y con la negación de ciertos saberes vinculados a su funcionamiento (como la ingeniería). En este sentido, las mujeres estaríamos desapropiadas de ciertos conocimientos particulares de la modernidad, como es el caso de las tecnociencias y, por lo tanto, desapropiadas de cierto tipo de "saber".

Siguiendo con este razonamiento, Judy Wajcman (1991) ofrece con el concepto de "generización de la tecnología" una argumentación de cómo todos los artefactos tecnológicos están conformados por las relaciones, los significados y las identidades de varones y mujeres, de tal suerte que la utilización de las tecnologías, así como su diseño, desarrollo y difusión, están estrechamente ligados a las jerarquías de género. 
Así, las armas y los automóviles se asocian con el uso masculino, mientras que los electrodomésticos y los anticonceptivos con el femenino. Esta lógica continúa con los avances tecnológicos computacionales de la segunda mitad del siglo XX, cuyos antecedentes se encuentran en los periodos de guerra, y particularmente en las armas de ataque, las cuales también han sido monopolizadas por los hombres. Así, a lo largo de los siglos, la tecnociencia se ha asociado con la construcción y control por parte de los varones (Wajcman, 2006).

Ahora bien, además del enfoque feminista, la investigación de la cual parte este artículo centra su atención en el campo de la tecnociencia, específicamente en la tecnología computacional, ${ }^{5}$ y para abordar esta esfera resulta necesario hacer referencia al surgimiento de la cibernética hacia los años cuarenta del siglo XX, pues es el antecedente inmediato del cómputo. La cibernética buscaba entender cómo piensan los seres humanos para luego hacer "pensar" a las máquinas e imitar los comportamientos de control de cambio y comunicación de los seres vivos (Wiener, 1948). Esta disciplina dio origen al cómputo moderno, el cual requiere que las máquinas sean capaces de seguir una serie de instrucciones ordenadas y finitas para ejecutar una acción, lo que hoy en día conocemos como algoritmos, y que luego fueron representados en el código fuente de un software.

Gracias al trabajo de la comunidad científica, particularmente de personas estudiosas de las matemáticas y la física, fueron creados los primeros programas de cómputo $^{6}$ en la década de los sesenta del siglo pasado, los cuales representan un importante cambio de paradigma en el desarrollo y ejecución de la técnica. El nacimiento del software marca, sin duda, el rumbo de la tecnología computacional que usamos hoy en día.

Para comprender mejor qué es el software o programa de computadora y cómo se relaciona con la técnica, se retoma la metáfora que usa Richard Stallman (2004), quien lo compara con una receta de cocina, ya que el software es un conjunto de recetas minuciosamente detalladas para la solución de un determinado problema, que puede ir desde hacer una suma hasta escribir una carta, crear un dibujo vectorial o editar un video.
Dichas recetas están escritas con sintaxis propias que son heredadas, al mismo tiempo, del inglés y de las expresiones matemáticas, a lo cual podemos llamar lenguajes formales, ${ }^{7}$ que son con los que están escritos los programas. La metáfora de la receta de cocina es usada también por Bernard Stiegler cuando vincula la técnica con la tecnología que integra la ciencia. Para este autor, la cocina se relaciona con la producción, con la transformación de materiales en productos y, por lo tanto, es fácilmente reconocida como técnica, es decir, como ya dijimos: saber-hacer (Stiegler, 2002).

Entrada la década de los setenta era muy común entre la comunidad de programadores del Instituto Tecnológico de Massachusetts (MIT por sus siglas en inglés) en Estados Unidos compartir softwarey, con ello, pedir y ofrecer parte del código fuente para mejorarlo colectivamente. Sin embargo, la lógica capitalista y el afianzamiento del neoliberalismo permearon en las prácticas computacionales de la época, y a finales de esa misma década algunas empresas pioneras de computación prohibieron el acceso al código fuente, su libre compartición, y tasaron un costo para la distribución de su software, a manera - exclusivamente- de programas ejecutables o aplicaciones (archivos binarios). Algunas computadoras modernas de la época comenzaban a tener su propio sistema operativo, para lo cual se necesitaba firmar un acuerdo de confidencialidad y obtener así una copia ejecutable (Stallman, 2004).

Paulatinamente, el uso y la compartición del código fuente se transformó en su privatización, de manera que se volvió cerrado y desembocó luego en la creación y uso de patentes de software en Estados Unidos. Muy poco tiempo después, la venta de software se volvió un negocio muy redituable para las corporaciones. Comenzó con ello la era de la comercialización del software sin código fuente abierto (lo que hubiera permitido conocerlo y estudiarlo); por el contrario, se trataba de un "código cerrado" que representó un velo y que impedía saber cómo fue hecho. Fue el inicio de la era del uso de una caja cerrada y desconocida como herramienta tecnológica. Si se toma en cuenta la definición de técnica en tanto saber-hacer, el cierre de código del software representa entonces "el nuevo ocultamiento y privatización del 
saber-hacer y otra forma de ejercicio de poder". Si la tecnología digital del siglo XXI que precisa el uso de software tiende a "ocultar" el código fuente, esto nos pondría necesariamente en un lugar cada vez más lejano de la técnica. Por lo tanto, la imposibilidad de ver y modificar el código es una problemática importante digna de reflexión y estudio, ya que el ocultamiento de la "receta de cocina" ofusca e impide también el estudio y el ejercicio de la técnica y de ciertos saberes.

Estas y otras implicaciones - como el hecho de que el software de código cerrado es propiedad privada - han motivado a un grupo de personas ${ }^{8}$ a llamarlo software privativo, para subrayar que "priva" libertades y "privatiza" el conocimiento. Lo cierto es que se ha convertido en un software comercial y hegemónico usado de manera muy habitual, normalizado, poco cuestionado y que replica los principios del sistema económico y político del capitalismo neoliberal.

Lo anteriormente desarrollado permite afirmar que la apropiación tecnológica computacional de las mujeres - en tanto articulación de prácticas, representaciones, valores compartidos (Martín, 2013) y procesos en torno al uso de los equipos de cómputo, que tienen como finalidad la integración de dicha tecnología y su transformación - se ve ofuscada no solo por el alejamiento de ciertas técnicas especializadas vinculadas con las máquinas y la modernidad en el capitalismo, sino que, además, en el caso de las tecnologías digitales y de información, se ocultan los procesos que lleva a cabo el equipo de cómputo - como programas que corren en paralelo sin que lo sepamos- y propician una mediación gracias al ocultamiento del código, el cual impide conocer cómo son hechos estos programas, qué hacen, para qué lo hacen y, sobre todo, imposibilita su modificación y adaptación. ${ }^{9}$ Si bien esto último afecta a todas las personas usuarias, la generización de la tecnología de la que habla Wajcman, y que fue mencionada anteriormente, sitúa a las mujeres en una esfera de alejamiento aún mayor.

Ahora bien, como consecuencia del cierre del código de programación y en contraposición a las prácticas de privatización que supone el software privativo, fue impulsado, entre 1983 y 1985, un movimiento social de resistencia que encuentra sus bases en la cultura y ética hacker: el movimiento de software libre.

El movimiento de software libre basa sus principios en la libertad de usar, estudiar, modificar y distribuir el código fuente, convirtiéndose en una posibilidad de conocer el interior de la caja cerrada y concebir el cómputo desde la perspectiva del código abierto, donde los "saber-hacer" y, por lo tanto, la técnica, están al descubierto; donde cualquier persona que pueda leer o estudiar el código y también modificarlo. Volviendo a la metáfora de la receta de cocina, la posibilidad de saber los ingredientes y los pasos para la elaboración permitiría contrarrestar la dependencia al software hegemónico, ${ }^{10}$ haciendo modificaciones para nuestros propósitos e intereses específicos, y con ello brindar una contrapropuesta al régimen capitalista de privatización. Además, el software libre está hecho de manera colaborativa y debe especificar expresamente en su divulgación que no se puede cerrar ni ocultar el código que se mejore para garantizar su permanente apertura.

El nacimiento del software libre sucede en el contexto de una cultura hacker cuya figura icónica no tiene que ver con el imaginario de "pirata informático" con el que popularmente se asocia, sino que data de la década de los sesenta y parte de la definición que la propia comunidad de hackers estadounidenses plasmaron en The Jargon File, un compendio de la jerga que define muchos de los aspectos de la tradición hacker, su folclore y su humor. En él se define a estas personas como quienes "gustan de programar de forma entusiasta" (The Jargon File, 2003) y para quienes es "deber de naturaleza ética compartir su competencia y pericia elaborando software gratuito y facilitando el acceso a la información y a los recursos de computación siempre que ello sea posible" (Himanen, 2002:5).

Aunque a lo largo de los últimos años las definiciones y caracterizaciones de las personas hacker han ido cambiando, incluso de manera negativa, para términos de este artículo es de relevancia subrayar que el espíritu de la actitud del hacker (lo que Pekka Himanen llaman la ética del hacker) es lúdico, y que sus motivaciones para resolver un problema se vinculan con la pasión por lo que hace, similar a la que se asocia con el arte y 
el trabajo intelectual, una actitud apasionada de goce y disfrute que se opone a la ética protestante del trabajo de la que hablaba Max Weber, surgida en el centro del espíritu capitalista del siglo XVII, y que tiene como ejes la vocación, el deber y el dinero (Himanen, 2002). Particularmente, es de mi interés el campo de las personas hacker que desarrollan habilidades computacionales especializadas a través de diversas prácticas gracias a que conocen el funcionamiento de su equipo de cómputo. Su ética, vinculada con sus prácticas especializadas, permite considerar la figura hacker como disidente en un mundo tecnológico normado, que disfruta de aprender los detalles de sistemas de programación con la capacidad de decidir diversos aspectos de la tecnología que usa, en contraposición con la mayoría de las personas usuarias que prefieren aprender únicamente lo mínimo necesario (The Jargon File, 2003). En este sentido, no es casualidad que en la comunidad hacker sea muy habitual el uso y la programación de software libre, ya que ambos son manifestaciones políticas que históricamente han ido de la mano.

Mientras que las y los ingenieros son figuras ponderadas en la modernidad que surgen para hacer funcionar las máquinas en la evolución del capitalismo, las personas hacker plantean otras formas de "saber-hacer". Tal como afirma Guiomar Rovira: "[el hacker] propone hacer ingeniería inversa para conocer cómo funcionan las máquinas que el mercado ofrece como cerradas, para darles otras terminaciones y usos [...] Es por ello que la figura del hacker se contrapone a la del ingeniero" (Rovira, 2017:110).

Así pues, el software libre, con raíces en la ética hacker, surge como una contrapropuesta que postula la apertura del código fuente para que todas y todos puedan modificarlo, y con ello aspirar a una generación de conocimiento más horizontal. Representa también un movimiento de resistencia tecnológico frente a las prácticas capitalistas neoliberales de privatización del conocimiento e intereses económicos.

A pesar de que el software libre, en el contexto de la ética hacker, cuestiona las formas de hacer y los valores del capitalismo, y aunque sus fundamentos son colaborativos y no competitivos, algunas de sus comunidades viven tensiones al estar inmersas en complejas relaciones de poder. Así pues, se observan relaciones de poder y meritocracias puntuales que pueden convertirse en jerarquías de larga duración como, por ejemplo, mantenedores ${ }^{11}$ de un desarrollo de software que deciden qué contribución de código entra y cuál no, o bien, que deciden no atender las contribuciones hechas por mujeres (Terrell et al., 2017) De igual forma, es evidente el desarrollo más veloz en los proyectos de software libre que obtienen más financiamiento o que les son más beneficiosos a la industria capitalista en la que se inscriben. ${ }^{12}$ Asimismo, el fundador del movimiento del software libre, Richard Stallman, ha sostenido en múltiples entrevistas la postura de que la ciencia es neutra y que, por lo tanto, el código también lo es, de tal suerte que no importa el género, la raza o la condición social de quien escribe el código (Haché, Cruells y Vergés, 2011; Natansohn, 2013).

La participación de las mujeres en el movimiento de software libre es considerablemente menor que en el de software privativo, ya que "sólo el 2\% de las personas que trabajan en el desarrollo de tecnologías son mujeres, mientras que en la industria del software propietario, el porcentaje es de 28\%" (Baroni, 2013:125). Si bien resulta paradójico que la participación de las mujeres que escriben código sea menor en un espacio aparentemente progresista, también es cierto que las reflexiones feministas han evidenciado que esto sucede en prácticamente todos los movimientos que pretenden la transformación social, aunque estos incorporen discursos de equidad de género. Asimismo, mujeres con identidades diversas, afrodescendientes, indígenas, transgénero, del área rural, etcétera, se encuentran ausentes o con poca participación.

En el contexto de este trabajo, las mujeres hacker son aquellas que desarrollan, diseñan y mantienen tecnología computacional dentro de las comunidades de software libre, mujeres en condición de excepcionalidad -y que gozan de ciertos privilegios, probablemente vinculados a su clase o raza- que a pesar del supuesto alejamiento tecnológico, marginalidad de saberes complejos y desvalorización del saber-hacer del que hablé al principio, aunado a su poca participación en este 
movimiento, escriben el código de programación que en otros espacios está cerrado y es secreto; es decir, estas mujeres hacker poseen un saber-hacer especializado, y ejecutan técnicas del siglo XXI.

\section{Verme para verlas: apuntes hacia una metodología situada}

Entrando al terreno de la propuesta metodológica, subrayo que este artículo parte de una investigación más amplia que es situada y que toma como uno de sus ejes metodológicos mi propia experiencia y mi devenir hacker, así como mi conocimiento situado (Harding, 2010), como parte del campo de investigación y desde la reflexión política de mi activismo en el movimiento mexicano de software libre desde hace diez años.

Cabe señalar que tanto el activismo como mi trabajo profesional me han llevado a participar en encuentros y eventos que me han permitido delinear y especificar, con mayor detalle, el perfil de las mujeres hackers a las que me interesa acercarme. Las reconozco a través de mi intención y necesidad de ser yo misma una mujer hacker, debido a mi inquietud por la problemática que representa el cierre de código de programación en la esfera social y, por supuesto, para el activismo feminista, que usa cada vez más los medios digitales como espacio de expresión y de lucha, que es objeto de múltiples capas de mediación tecnológica, así como de violencias en la virtualidad.

Mi estrategia metodológica se ha entretejido con el avance de la investigación - y sigue en construcciónya que uno de los aprendizajes que se incorporaron a este proceso fue el de reconocer mis prácticas, es decir, verme a mí, situada en mi propio campo de estudio, hacer consciente mi devenir hacker y reconocer a mis interlocutoras a través de la admiración y el propósito de "querer ser ellas", de convertirme en una mujer hacker frente a la urgente necesidad de vincular la lucha feminista con la disidencia tecnológica, en el marco de una violencia flagrante contra las mujeres en el ámbito digital y de una vigilancia y control masivo por parte de las corporaciones tecnológicas. Así pues, la búsqueda de estas mujeres se conduce por mi propio devenir hacker, es decir, son mujeres a las que voy encontrando y reconociendo en mi proceso.

Acudir a diversos encuentros nacionales e internacionales de las comunidades de software y cultura libre, como el Internet Freedom Festival o el Creative Commons Summi, ${ }_{13}^{13}$ así como el "Encuentro Hackfeminista: tecnología y afectos”, realizado en San Cristóbal de Las Casas, Chiapas, en julio de 2019, entre otros, ha sido una forma de encontrarlas. Para establecer el primer contacto con ellas, fue necesario generar vínculos de confianza reforzados por personas conocidas del campo, el reconocimiento de los trabajos realizados, así como el mutuo conocimiento de nuestras trayectorias profesionales y activismo.

A partir de una primera exploración del campo, la cual se realizó desde finales de 2018 y a lo largo de todo el año 2019, fue posible el encuentro con mujeres desarrolladoras y sys-admin, ${ }^{14}$ quienes fueron las primeras interlocutoras que posteriormente me fueron conectando con otras más. Así es como hasta el momento de la elaboración de este artículo he logrado acercarme a y entrevistar a ocho mujeres hacker de diversas latitudes, edades y condiciones sociales.

Como parte del proceso para mapear el campo de estudio, y para reconocer el uso de tecnología computacional en mujeres, realicé un esquema (ver Figura 1) que parte de mi propia experiencia e inmersión al formar parte del campo. En él se pueden observar cuatro aros que representan cuatro diferentes niveles del uso que dan las mujeres a sus computadoras, que van de arriba hacia abajo, del más amplio con un mayor número de mujeres, al más reducido en tanto el porcentaje es menor. Estos niveles se organizan de la siguiente manera:

1) En el primer aro, el más amplio, se encuentran las mujeres que no utilizan computadoras o lo hacen de manera poco frecuente, quizá motivadas por familiares cercanos para mantener cierto tipo de comunicación. El equipo de cómputo que usan es el celular y algunas de ellas lo harán solo para hablar por aplicaciones de mensajería instantánea. Este aro será denominado: "mujeres con negación al uso" y responde a mujeres de más de 60 años, de clase media o media baja. 
2) En el segundo aro, en orden descendente, se encuentran las mujeres que usan tecnologías digitales como auxiliares en la realización de tareas cotidianas en la casa y la oficina, usan redes sociodigitales e incluso podrían realizar algún tipo de ciberactivismo; también manejan equipos de cómputo en tanto usuarias promedio de aplicaciones y programas para generar objetos digitales como textos, documentos, fotos y videos. Se trata de "usuarias con cierta experiencia" en el uso de tecnologías de cómputo, con edades de entre 40 y 60 años, de clase media.

3) El tercer aro, que a su vez disminuye en tamaño pues las mujeres que en él se encuentran son un menor número, incluye a las hackers-pre coders, ${ }^{15}$ a las que avanzada esta investigación he llamado hackfeministas, ${ }^{16}$ debido a que algunas se han autonombrado y han sido reconocidas así por sus pares. Yo me posiciono y sitúo en este aro. La mayoría de las ubicadas en este nivel somos usuarias de software libre, con un mayor dominio y conocimiento de la tecnología digital que usamos, y nos relacionamos de manera particular con nuestros equipos de cómputo. Hay nociones y preocupaciones por temas como la seguridad digital y el anonimato, así como una intención constante de cuidar y vigilar las prácticas computaciones (navegar en Internet de manera anónima, evitar la instalación de aplicaciones de redes sociodigitales, cuidados digitales, uso de correo cifrado, etcétera). Durante el proceso de esta investigación, y después de diversos encuentros entre colegas hackfeministas, puedo decir que por nuestras prácticas y la labor educativa y de divulgación que realizamos, muchas quienes nos encontramos en este aro fungimos como una especie de "puente" entre las colectivas feministas que realizan activismo en redes sociodigitales y temas técnicos de cómputo que pueden sonar complejos. Hemos realizado, al menos una vez, acompañamiento en casos de violencia digital, o bien asesorías sobre seguridad en Internet; cuestionamos el uso de redes sociodigitales privativas como Facebook y Twitter para el activismo, y no las usamos de manera frecuente. Oscilamos entre los 28 y 40 años, de clase media.
4) Por último, en el aro más pequeño y que representa una especie de "profundidad" de conocimiento y prácticas, se encuentran las mujeres hackers, a las que he llamado también hackers-coders, cuyo nivel de experiencia y dominio de la técnica es infinitamente mayor al promedio. Son programadoras, administradoras de sistemas computacionales, desarrolladoras web o mantenedoras de código fuente. Usan de manera restringida las redes sociodigitales, o bien, no cuentan con un perfil en estos software como servicio, ${ }^{17}$ de igual forma, manejan identidades anónimas en Internet, usan correos cifrados y suelen ser sumamente críticas sobre los servicios que ofrecen los grandes corporativos tecnológicos como Google, Amazon, Facebook, Apple y Microsoft. Como ya he mencionado, las mujeres ubicadas en este aro constituyen el interés central de este trabajo. He encontrado que son de clase media o media alta, y sus edades varían, pues hay mujeres de 50 años, pero también de 30 .

Como mencioné anteriormente, durante el proceso de este trabajo, así como para el análisis y la realización del esquema anterior, tuve que ubicarme y reconocerme en uno de esos aros (ver Figura 2), lo cual no solo guió la reflexión que ha ido moldeando este proyecto, sino que me permitió reconocer mis propias prácticas, visibilizarlas y re-apropiármelas, como parte de un conocimiento personal que luego me permitió abrir otros enfoques y conectar de otra manera con las interlocutoras, e incluso incluirlo en los talleres y clases que he ido impartiendo en el camino. Considero que esto enmarca uno de los compromisos centrales de la investigación feminista: "el cambio para las mujeres en particular y el cambio social progresivo en general" (Blázquez, Flores y Ríos, 2012).

Autorreconocerme como "puente" en este proceso ha sido fundamental para el acercamiento a las interlocutoras y para ubicar el contexto del campo de estudio en tanto dinámicas sociales. Para sustentar esto, retomo lo propuesto por Helen Longino y su "empirismo contextual" (Longino en Blázquez, Flores y Ríos, 2012) que, en el marco de la epistemología feminista, propone tratar el conocimiento social como elemento de análisis objetivo a través de respuestas comunitarias, compar- 
tición y equidad de autoridad intelectual, lo cual fue fundamental en el acercamiento con las interlocutoras, pues "nadie sabe más que otra", sino que partimos de experiencias distintas. La intención es no separarme de las sujetas de estudio, no tomar excesiva distancia y evitar con ello la deshumanización, y no tratarlas o verlas como objetos. Asimismo, la "objetividad fuerte" de Harding, en la que todas las "fuentes de error o de prejuicio tanto cultural como técnico sean tomadas en cuenta" (Blázquez, Flores y Ríos, 2012:28), es otro de los sustentos teóricos de mi intención de "situarme" en el campo de estudio y de reconocer mis posibles sesgos en el tema, y más que enfocarlos como algo "negativo" o negarlos, trabajar desde ellos, reconocerlos y usarlos con conciencia.

Me parece importante mencionar, como parte de una posible metodología feminista, el hecho de que este proceso de investigación me ha permitido no solo reconocer a mis interlocutoras, sino también ver mis propias prácticas y mi proceso de apropiación tecnológica que, como mencioné anteriormente, buscará la integración a la vida y la transformación de dichas tecnologías, lo que eventualmente me ayudó a reconocer un "devenir hacker" que me ha posibilitado observar, interesarme y ser más incisiva en las prácticas de mis interlocutoras. Mi propio camino ha servido como lente para la observación del campo, porque de igual manera este devenir hacker se define en gran medida por las prácticas y los "saber-hacer" que realizamos (ver Figura 3), qué se hace, cómo se hace y qué sentido se da a esas prácticas.

Es por ello que considero que existe una dinámica dialéctica del devenir hacker y del "saber-hacer", es decir, que se "sabe" a través de las prácticas (o sea, del hacer) y se "hace" a través de los saberes que se generan. El saber-hacer influye en el devenir hacker y viceversa.

\section{Conociendo a las hacker: primer acercamiento}

A partir de esta reflexión y "lente" para la aproximación al campo, cabe señalar que lo que desarrollaré a continuación no es propiamente el primer acercamiento - pues he formado parte de él desde hace algunos años-, pero sí se trata de las entrevistas primeras en que realizo una batería de preguntas que me permiten entablar un diálogo con las interlocutoras y conocer la manera en la que han aprendido lo que saben, cómo han adquirido sus conocimientos y qué significa esto para ellas. También es importante mencionar que este trabajo sigue en proceso, por lo que incluso podrá distinguirse apenas una labor descriptiva que da cuenta de un panorama general que abre incluso nuevas preguntas.

Realicé las entrevistas del 13 de noviembre de 2019 al 15 de febrero de 2020, aunque, como mencioné, ya había realizado la búsqueda de las interlocutoras y pactado esta comunicación a finales de 2018. De las ocho entrevistadas, seis de ellas tienen entre 30 y 36 años de edad, y las dos restantes tienen 40 y 50 años, respectivamente. Cinco son mujeres latinoamericanas, dos de ellas se consideran mestizas y de la periferia de la Ciudad de México, la mayoría tiene estudios universitarios y se consideran de clase media (ver Cuadro l).

\section{Procesos de aprendizaje: aprender como forma de resistencia}

Uno de los temas que se abordaron en las entrevistas fue el de los procesos de aprendizaje: cómo aprendieron o cómo llegaron a obtener conocimiento. Esta pregunta resulta importante no solo para conocer sus caminos y su eventual devenir hacker, sino también para indagar y tratar de probar la hipótesis de trabajo, que probablemente aprenden de sus contrapartes masculinas, figuras hackers respetadas por la comunidad, que suelen ser sus parejas sentimentales.

Las respuestas de todas coinciden en que, a pesar de tener estudios en alguna carrera vinculada con las TIC o ingenierías, o bien cursos formativos en estos temas, mucho o gran parte de lo que saben lo adquirieron gracias a que son autodidactas y han "aprendido bajo la marcha”. Esto coincide fuertemente con las caracterizaciones de la figura del hacker que proponen tanto Himanem (2002) como Levy (1984). La mayoría de las entrevistadas coinciden en que han aprendido gracias a la resolución de problemas, a que buscan en manuales o preguntan en foros y a otras personas. Tal como apunta Maadix:18 "la forma en que más aprendí es autodidacta, o sea, luchando, rompiéndome los cuernos contra un 
problema concreto" o "aprender a hacer algo para luego poder enseñarlo a otras personas", como dice Karen, antropóloga social que hoy da clases de programación para la infancia y que afirma haber aprendido haciendo tareas por sí misma, a fuerza de prueba y error. Esto se conecta directamente con lo que afirma Himanem en su icónico libro La ética del hacker y el espíritu de la era de la información, en el cual afirma que: "El proceso de aprendizaje característico del hacker empieza con el planteamiento de un problema interesante, sigue con la búsqueda de una solución mediante el uso de diversas fuentes, y culmina con la comunicación del resultado para su exhaustiva composición" (Himanen, 2002:59).

La educación "no formal", característica del aprendizaje de las personas hacker, es señalada también por McKenzie Wark, cuando hace referencia al concepto de educación: "La educación no es conocimiento. Tampoco es el medio necesario para adquirir conocimiento. El conocimiento puede surgir con igual facilidad de la vida cotidiana. La educación es la organización del conocimiento dentro de las restricciones de la escasez, bajo el signo de la propiedad" (McKenzie, 2004:36).

Para Himanen, "la actividad del hacker es también gozosa. A menudo se enraíza en exploraciones lúdicas" (2002:14), afirmación que coincide en gran medida con los testimonios de todas las entrevistadas; por ejemplo, cuando le pregunté a Maddix acerca de lo que significaba para ella programar, respondió: "iA mímeencanta! Osea, creo que es algo muy divertido porque tienes que clarificar cómo solucionar algo". La mayoría de las entrevistadas coincide en que les resulta divertido, que parece mágico, pero que no lo es, esto porque lo han hecho ellas, en gran parte, en un entorno de juego:

Ese verano yo me aburría y ya me conocía todos los juegos, así que empecé a leer los libros que venían con el ordenador, sobre programación en iBasic!, a escribir el código que venía en el libro y el ordenador me decía: "Hola Juga!" =D, me parecía mágico, seguí leyendo y empezando a entender... era mucho más divertido que los juegos e intuía la de cosas que se podrían hacer (Entrevista a Juga ).
Ahora bien, algo que toma particular relevancia en términos de género es que las entrevistadas coinciden en la importancia de aprender para hacer las tareas por sí mismas, solas, sin la necesidad de pedirle a un hombre que lo solucione, mencionan, y hacen alusión constante a la emoción que les implica resolver tareas solas y ser más autónomas e independientes: "Si quieres hacer algo, aprender por ti misma [...] si puedes hacerlo tú, mejor hazlo tú". Otra de las entrevistadas explicó:

Y, claro, me encontraba con esto de que te dicen: "Ah, pero esto no se puede hacer" [tono burlón] a ver que la típica... Y isabes? yo: “Cómo que no", yo trabajaba en la web de mi entorno virtual, tenía una máquina virtual, configuraba mi Apache, luego lo probaba y me decían: "No, que esto no sé qué, no sé cuánto", tratándote como que... isabes? "Es laúltima pringada”... Y esa fue también una razón por la que dije: "Hostia, yo... quiero aprender, o sea, también programar, y no saber nada del entorno sobre que corran las aplicaciones, no, no puede ser" [...] Sí, que fue un poco así, de querer también tener más independencia, no tener que pedirle al maromo en turno que te haga algo, isabes?, y saber hacértelo tú, o si no te lo puedes hacer tú, ipor qué lo tienes que pedir? Porque el entorno es tal que no puedes acceder, o sea, isabes? Pues saber contestar a tono de decir: "A ver, que no lo sepas hacer no quiere decir que no se puede hacer", Pues eso... (Entrevista a Maddix).

Esto, a la par de lo que expresan otras entrevistadas, como Paola Villareal, hacker mexicana, considerada una de las cien mujeres más influyentes de 2019 por la $\mathrm{BBC}$, quien coincide en que no todas las personas están dispuestos a enseñar: "[Fui] preguntando en los chats, aunque ahí te encontrabas con gente que no necesariamente quería compartir conocimiento, hasta que te toparas con alguien que sí, pero era cosa de preguntar [...] fue mi primer ejercicio de aprender a aprender, pues no todo el mundo quería explicar".

Esta conjunción entre la posibilidad de hacerlo por sí mismas en tanto proceso de independencia, y el obstáculo de que no siempre hay alguien dispuesto a enseñar, se enlaza con una de las afirmaciones de Verónica 
Valeros, especialista argentina en ciberseguridad, quien afirma que al trabajar en estos entornos "no dependes de otra gente para aprender, eso es una ventaja cuando eres mujer, porque luego los profes no te quieren explicar".

En este sentido, March, mujer guatemalteca que hoy reside en la Ciudad de México, retomó la idea de que el sistema capitalista nos ha quitado también la posibilidad de adquirir conocimientos y afirmó: "es que no hay tiempo para aprender". Esto me lleva a pensar que quizá para estas mujeres el proceso de aprendizaje se vuelve una práctica política, una especie de acto de resistencia, que da cuenta de un camino recorrido en tanto su condición de género. March y yo hemos dicho al unísono en la entrevista "aprender como acto de resistencia”.

Ahora bien, el tema del proceso de aprendizaje en materia de saber-haceres computacionales también se conformó con la pregunta: icuál es tu primer recuerdo con un equipo de cómputo $?^{19}$ Esta llevó a respuestas vinculadas con el entorno en que se desarrollaron sus intereses, así como sus facilitadores y trayectorias, tal como sucede en la investigación de Donastech (Vergés, Cruells y Haché, s/f).

En este sentido, es de particular interés que todas las entrevistadas coinciden en que tuvieron una computadora en casa, adquirida por padres, madres o tías, y que, en algunos casos, nadie de su familia sabía usar el equipo de cómputo, lo cual les daba la libertad de utilizarlo durante mayor tiempo y con pocas restricciones.

La primera computadora que yo tuve fue cuando yo tenía ocho, que un vendedor vino a vender computadora y mi mamá le compró una [...] creo que tomó un rol muy importante que mis papás no sabían cómo usar la computadora [...] a otros amigos que tenían padres que sabían más de computación, nunca se acercaron tanto porque siempre había otra persona que les arreglaba o les corregía o les resolvía los problemas. Creo que gracias a que para mi padre la tecnología también era nueva, no sabía de tecnología, quizá no le ponía género, y ya le costaba un poco aprender, así que no decía aquello de "dejame a mí, que tú no sabes", y me dejaba que manejara la tecnología (entrevista a Juga)
Por otro lado, algunas otras entrevistadas mencionaron que fue una mujer quien las inició en estas prácticas, como en el caso de Karen, cuya tía, que había estudiado una licenciatura en Informática, le conminó a usar la computadora que llevó a casa, sin temor: “tócala sin miedo", le dijo. Por su parte, Martha también usó la computadora por primera vez impulsada por su madre, quien era ingeniera en sistemas. Maadix aprendió a programar siguiendo los pasos de su madre, que también fue programadora en la década de los ochenta.

Aunque aún es una afirmación por trabajar con mayor detenimiento, puedo decir en este punto de la investigación que estas mujeres hacker, quienes tienen un alto dominio de la técnica, no tuvieron figuras masculinas que les impidieron pasar tiempo frente a la computadora, ya sea hermanos mayores a los que se les permitiera usarla mayor cantidad de tiempo solo por el hecho de ser hombres o padres que acapararan el uso del equipo de cómputo como parte de sus actividades cotidianas. El factor de la edad es también un aspecto importante de análisis, ya que las más jóvenes señalaron que "aprendieron de compañeras y no tanto de mentores" o "prefieren preguntarle a amigas", sobre todo las que han trabajado en ambientes colaborativos feministas como March o Verónica. Esta última apuntó: "aprendí más por mi cuenta, tuve pocos mentores". De igual manera, hay un elemento de clase que marca la posibilidad de pasar tiempo frente a una computadora sin que la necesidad de hacer labores de cuidados se los impida.

Las más "técnicas", como Paola, líder de proyecto en el CONACyT en México, Juga y Hiro del proyecto Tor o Maadix de Xnet coinciden en que nadie de su familia sabía usar una computadora, sus padres se encontraban ausentes o simplemente nadie tenía idea de lo que hacían frente al nuevo aparato, por lo que tuvieron libertad de experimentar y aprender. Por supuesto, no podemos olvidar las categorías de clase, raza y localización geográfica. Juga, Hiro y Maadix son mujeres del norte global, blancas y de clase media; Paola es mexicana, blanca, de clase media, con estudios de bachillerato. 
Por último, a pesar de que varias de ellas tuvieron un acercamiento a una computadora a temprana edad, tal como lo apunta Donastech, "resulta inquietante el vacío temporal que presentan muchas de sus experiencias en el tiempo. Después de este primer contacto, muchas no son animadas, empujadas o alentadas a injerirse en lo tecnológico" (Vergés, Cruells y Haché, s/f :8). Estas mujeres obtuvieron algún tipo de formación formal, ya sea en cursos, orientaciones o programas en preparatoria o secundaría, lo cual coincide con la afirmación de algunos informes de la UNESCO, donde consta que quienes estudian asignaturas vinculadas con ciencia, tecnología, electrónica y matemáticas (STEM) tienen muchas más posibilidades de avanzar en programas universitarios en estas mismas áreas (UNESCO, 2019). Asimismo, hay que apuntar que, si bien abandonaron estas inquietudes, luego las retomaron cercanas a la época universitaria, en su mayoría motivadas por el uso de tecnologías como el software libre.

\section{Prácticasy saberes}

Algunas autoras hablan de las implicaciones del código en tanto materia prima que produce y genera saberes (Baroni, 2013). Sin embargo, uno de los primeros hallazgos al entrevistar a estas mujeres es que, más que "hacer código", les resulta interesante y emocionante "lo que se hace con el código", tal como apuntó Maadix:

Entonces, la parte luego de escribir código es lo de menos, o sea, la parte más divertida es diseñar... ino? la solución en abstracto, sin que, da igual el código en el que lo implementes, entonces es la capacidad de, o sea, de estar pensando, es algo que... puedes trabajar casi sin pensar, solo razonando antes de irte a dormir, y por la mañana te despiertas con la solución... [risas]. Quiero decir, es la parte divertida y no monótona de algo que tienes que investigar. O sea, encontrar soluciones que tienes que adaptar a retos que tengas en este momento... Luego, el código en práctica y ver que la idea que has tenido efectivamente servirá. Entonces, a mí me gusta mucho porque esto es como una, una ciencia, digamos, no falla, y si falla es porque hay un error, ime explico? (entrevista a Maadix).

Existe también una relación con emociones vinculadas al entusiasmo y la capacidad de pensar en "macro", en diseñar, razonar, resolver retos:
Me encanta, cuando estoy inspirada lo hago rapi- dísimo y es superemocionante para mí resolver un problema, pero más que hacer código, me gusta pensar en los sistemas desde una visión más macro, más de arquitectura y estrategia, para hacer eso tienes que saber hacer sistemas, modelar datos, generar una estrategia de desarrollo (entrevista a Paola).

Así, planear, pensar, crear estrategias para solucionar problemas y diseñar para resolver un reto en particular, resultan ser prácticas muy motivantes para estas mujeres, más allá de hacer código por sí mismo. Esto puede resultar particular de las mujeres hackers, ya que en muchos entornos de colaboración de software se busca que el código sea "limpio", casi perfecto, haciendo alusión a que el código puede ser también una obra de arte (Cox y McLean, 2013), además de que resulta imprescindible hacerlo bien y limpio, pues será visto por varios ojos, además de que será sometido a escrutinio y probablemente a críticas (Piñeiro, 2003).

Siguiendo con la idea de escribir código para un fin, una de las interlocutoras hizo mención de algo que me parece particularmente interesante por estar vinculado con una reflexión social, pero también articulado con un autoconocimiento personal:

Creo que lo que me resulta especial en escribir código es que se puede aplicar a áreas muy diversas. Por ejemplo, se puede escribir código para relacionar conflictos políticos en el mundo. No sé cómo sería yo sin escribir código, después de más de 20 años haciéndolo. Si no escribo código en varios días, es difícil encontrar cosas que [me] entretengan y empiezo a pensar en algo que programar. A veces viendo el código que he escrito en otros momentos, me doy cuenta de cuál era mi grado de atención o incluso estado de ánimo. O cómo no 
sabía de ciertas prácticas que sé ahora, o en cambio tenía mucho más dominio de temas que ahora he olvidado (entrevista a Juga)

La particularidad de "conocerse a través del código" fue mencionada únicamente por una de las entrevistadas, la misma que vinculó procesos de aprendizaje al compartir su código: "aprendo mucho de los comentarios que otras hacen sobre mi código". Me parece algo sumamente interesante para articular el significado que le dan ciertas mujeres hacker a sus prácticas, con lo que menciona Wajcman sobre la agencia de saberes particulares de mujeres en las prácticas sociotécnicas (Wajcman, 2006).

Resolver proyectos propios con programación, automatizar procesos, usarlos para comunicar, hacer código para solucionar problemas y hacer código para realizar proyectos divertidos son algunas de las ideas que se repiten entre las entrevistadas al momento de hablar de su práctica con el código, lo cual me lleva a la pregunta de si no será que una de las particularidades en tanto su condición de género es el contacto con lo "útil" socialmente, con un pensar en otras personas, sin dejar de lado que sea algo que las entusiasme y les genere emoción o placer al hacerlo, lejos de la necesidad de "probar" o "legitimarse" con sus prácticas, como sucede con sus contrapartes varones.

\section{Conclusiones: primeros hallazgos, nuevas preguntas}

Para estas mujeres hacker, hacer lo que les divierte, resolver retos, la curiosidad, la experimentación y el factor lúdico son fundamentales tanto para su aprendizaje como para su vida profesional, lo cual concuerda con el perfil que varios autores han descrito para referirse a la figura hacker. Sin embargo, quizá una particularidad de estas mujeres es el hecho de que lo hacen también como un acto de independencia y autonomía; para demostrarse que pueden hacerlo por sí mismas, sin la ayuda de un hombre. Esto, aunado a que lo hagan aún con toda la serie de obstáculos propios de su condición de género, representa un logro aún mayor, por el que se sienten orgullosas, aunque en algunos casos "no termina de ser suficiente". Por supuesto, esto también depende del factor social de clase.

Lo anterior abre la reflexión hacía nuevas preguntas: hacer lo que les satisface es una práctica del hacker, pero en el caso de las mujeres iexiste algo distinto que no necesariamente se vincule con la necesidad de ser validada por sus pares? El hecho de que hacer código de programación no les resulte tan significativo tanto como lo que hacen con él ies una condición de género que las vincula al bien comunitario, a una conexión social, a resolver un problema en la vida "real"? Hasta ahora se ha probado falsa mi hipótesis de que hay varones detrás de la forma en la que accedieron al conocimiento o en sus procesos profesionales. Esto quizá se pruebe cierto en un "aro" anterior en mi tipología empírica del uso de la tecnología en mujeres (ver Figura 1), y solo se observa en las hackfeministas. Por el contrario, las mujeres hacker con este nivel de dominio de la técnica se abrieron paso gracias a que no había una figura masculina que obstaculizara sus exploraciones con el equipo de cómputo; sin olvidar, por supuesto, su condición de clase, raza y localización geográfica. Algunas de ellas, como Karen o Martha -mujeres jóvenes - o Maadix de 50 años, no contaron con una figura masculina, pero sí femenina (tía y madre), que les abrió el camino para el devenir hacker.

Observé también algunas tensiones que merece la pena afinar más, como el hecho de las contradicciones entre la emoción de sentir que lo han hecho por sí mismas, pero al mismo tiempo no confiar en que puedan obtener una certificación o mantener un servidor web. De igual forma, observé la tensión entre saber que son tratadas de manera distinta por ser mujeres, pero a la vez "no pensar mucho en eso" y en ocasiones querer hacerse pasar por hombres. Por otro lado, las mujeres que parecen no sentir el llamado síndrome de la impostora, del cual se habla de manera recurrente en encuentros de tecnología, y con que el que me he identificado durante mi proceso profesional, coinciden en que son las que gozan de ciertos privilegios, que tienen mayor edad y experiencia, pertenecen a clases medias, y son blancas o del norte global. 
Por último, aunque no menos importante, se debe apuntar que todas coinciden en la relevancia de aprender de los errores, de solucionar problemas y de la capacidad de experimentar, de explorar y de aprender con base en prueba y error. Esto resulta interesante porque se contrapone a la idea de "inmediatez" y "facilidad" que ofrece la tecnología, asociada a productos que se comercializan, que son fáciles de usar y asequibles para (casi) todas las personas - que puedan pagarla-, como aplicaciones del celular. Aquí cabe preguntarse si no será que una posible tecnología que se oponga al control, o que permita adquirir cierto nivel de autonomía, apropiación e independencia, no tiene que ser necesariamente "fácil", sino amable y cercana a los procesos y ciclos de experimentación y aprendizaje. Si partimos de lo que ha mencionado Rivera Cusicanqui (2018) sobre los "saberes" que nos ha quitado la modernidad, el capitalismo, en tanto que ya no creamos nada con nuestras manos, lo que nos lleva a desconocer ciertos procesos, iserá que la tecnología que lleve a un posible devenir mujer hacker, y que a su vez permita fisurar los mecanismos de control, apropiación y extracción de datos, a partir de una propuesta hackfeminista, no tiene que ser necesariamente fácil?

\section{Notas}

${ }^{1}$ Para Stiegler, en el concepto tecnociencia: "las técnicas y ciencias se hacen inseparables, la racionalidad está sometida a la utilidad. Para Habermas, la utilidad del capital como 'actividad racional respecto a un fin' es una inversión, incluso una perversión del modelo epistemológico inicial de la filosofía en el que la teoría, esencia de la ciencia, se define por su independencia con respecto a finalidades útiles, es decir, antropocéntricas" (Stiegler, 2002)

2 El código fuente es un conjunto de líneas de texto que son las instrucciones que debe seguir la computadora para ejecutar un programa, y por lo tanto, realizar ciertas acciones. Así, en el código fuente de un software está descrito por completo su funcionamiento y está escrito en algún lenguaje de programación. El filósofo Pekka Himanen lo define como "el ADN de un software, su forma en el lenguaje utilizado por los programadores para desarro- llarlo; sin el código fuente, una persona puede utilizar un programa, pero no puede desarrollarlo en nuevas direcciones". En este protocolo de investigación, hablaré del código fuente y del "código" de manera indistinta.

${ }^{3}$ Siglas para designar a las cinco empresas más grandes de tecnología: Google, Amazon, Facebook, Apple y Microsoft.

${ }^{4}$ La primera vez que escuché el término "saber-hacer" fue en un taller en 2006, donde conocí el software libre, en el Laboratorio Arte Alameda en voz de un hacker. Años después, algunos grupos de investigación, como el conformado en la Facultad de Filosofía y Letras de la UNAM, identificado en Twitter con el hashtag \#SeminarioTF, lo han usado también en un sentido más amplio. Me parece importante mencionar el origen de la categoría en mi vocabulario, al ser recurrente en el discurso, además de ser muy usado en la cultura hacker.

${ }^{5}$ En la industria es habitual el nombre de TI (Tecnologías de la Información) para referirse específicamente a este campo; sin embargo, para términos de esta investigación se considera necesario el uso del término Tecnologías Computacionales para no referir necesariamente a la industria del cómputo y al campo empresarial y vincularlo también con el ámbito social.

${ }^{6}$ Programas de cómputo y software serán términos usados como sinónimos en este trabajo.

${ }^{7}$ Ejemplos de lenguajes formales de programación son: Phyton, C++, Java, Javascript, Ruby, Cocoa, Cobol, etcétera.

${ }^{8}$ Es importante señalar que este grupo de personas fueron usuarias del sur de América Latina, particularmente en Argentina; fue el filósofo Enrique Chaparro quien acuñó el término: software privativo. Lo dice Richard Stallman en entrevista en 2006, la cual puede consultarse en el siguiente link: https://www.consumer.es/tecnologia/software/richard-stallman-padredel-software-libre.html

${ }^{9}$ Hay muchas otras problemáticas incluso dentro de un código abierto. Estas problemáticas pueden ir desde no comentar el código que se realiza, lo que impide que otras personas conozcan los procedimientos y el idioma, hasta una complejidad importante por la cantidad de datos que se manejen. La apertura, en términos re- 
cientes, no es condición suficiente para la apropiación tecnológica, aunque sí necesaria. Tiene que ver con diversas formas hegemónicas de hacer ciencia.

${ }^{10}$ Cabe señalar que los desarrollos de ciertos software libre populares son producto del impulso y financiamiento de empresas privadas, como Blender, que obtuvo un fuerte financiamiento de la industria cinematográfica estadounidense.

${ }^{11}$ Un mantenedor o mantenedora de código es una persona autora o coautora de un software que acepta o rechaza contribuciones al código. Generalmente, un mantenedor es el autor del programa, aunque en otras este abandona su desarrollo.

${ }^{12}$ Un ejemplo de ello es el programa de modelado y animación 3D llamado Blender, el cual es software libre y tuvo un desarrollo muy veloz debido al financiamiento de la industria del entretenimiento estadounidense, particularmente del cine y los videojuegos.

${ }^{13}$ Global Summit Creative Commons es la Cumbre Global del movimiento en pro de los comunes, también conocido como bienes comunes.

${ }^{14}$ Término en inglés que significa: "administración de sistemas", se refiere a la administración, mantenimiento e instalación de un servidor web.

${ }^{15}$ Coders es el nombre en inglés que se otorga de manera coloquial a las personas que hacen código. Fue el término usado en la primera estructuración de esta investigación. He decidido conservarlo para explicar que, aunque las mujeres de este aro tienen un nivel más amplio de conocimiento, no hacen código de manera regular o cotidiana.

${ }^{16}$ Para una investigación más amplia de esta categoría y caracterización del grupo, revisar el trabajo de la maestra Fernanda Briones, quien ha realizado entrevistas a diversas colegas. La entrevista que me realizó para su tesis doctoral (que se encuentra en curso) me ayudó a situarme y reconocerme en este aro propuesto.

${ }^{17}$ El software como servicio, en inglés software as a service (SaaS), es un modelo de negocio en el que las compañías tecnológicas ofrecen alojamiento de datos y soporte en un servidor a los que se accede vía Internet. Empresas como Google o Facebook se encargan de mantener y operar servicios diariamente para que las personas usuarias tengan acceso desde cualquier computadora. Las personas usuarias pagan estos servicios con sus datos personales, historiales de navegación y hábitos de consumo, entre otros.

${ }^{18}$ Los nombres de las entrevistadas responden a cómo ellas quieren ser nombradas.

${ }^{19}$ Decidí modificar la pregunta frecuente en otros trabajos de investigación similares que utilizan la frase "tu primer contacto con la tecnología" para invocar recuerdos de la infancia que se vinculen también con su noción de "tecnología", no necesariamente de una computadora. Sin embargo, es de mi interés hablar directamente de la relación de las entrevistadas con un equipo de cómputo.

\section{Referencias}

Baroni Selaimen, Graciela (2013). “Mujeres desarrolladoras de tecnologías: El desafío de las historias invisibles que viven entre ceros y unos". En Graciela Natansohn (coord.), Internet en código femenino. Teorías y prácticas. Buenos Aires: La Crujía Ediciones, pp. 123-136.

Blázquez Graf, Norma, Fátima Flores Palacios y Maribel Ríos Everardo (coords.) (2012). Investigación feminista: epistemología, metodología y representaciones sociales. México: Centro de Investigaciones Interdisciplinares en Ciencias y Humanidades, Centro Regional de Investigaciones Multidisciplinarias-Universidad Nacional Autónoma de México.

Boix, Montserrat y Ana de Miguel (2002). "Los géneros en la red: Los ciberfeminismos. El ciberfeminismo social". En Virgilio Almeida, Ricardo Baeza Yates y María Inés Camarena (eds.), The Role of Humanity in the Information Age: an Ibero-American Perspective. Santiago: Universidad de Chile.

Cox, Geoff y Alex McLean (2013). Speaking Code: Coding as Aesthetic and Political Expression. Cambridge: MIT Press. Cruells López, Eva, Núria Vergés Bosch y Alex Haché (2014). "Activismo feminista 2.0". En Pueblos. Revista de Información y Debate, 6 de junio. Disponible en http:// www.revistapueblos.org/blog/2014/06/06/activismo-feminista-2-0/

Federici, Silvia (2004). Calibán y la bruja: Mujeres, cuerpo y acumulación originaria. Madrid: Traficantes de Sueños. 
Haché, Alex, Eva Cruells López y Núria Vergés Bosch (2011). Mujeres programadoras y mujeres hackers. Una aproximación desde Lela Coders. S.p.i. Disponible en http:// www. rebelion.org/docs/141550.pdf (consultado el 3 de marzo de 2013).

Harding, Sandra (2010). “iUna filosofía de la ciencia socialmente relevante? Argumentos en torno a la controversia sobre el punto de vista feminista". En Norma Blázquez Graf, Fátima Flores Palacios y Maribel Ríos Everardo (coords.), Investigación feminista, epistemología, metodología y representaciones sociales. México: Centro de Investigaciones Interdisciplinares en Ciencias y Humanidades, Centro Regional de Investigaciones Multidisciplinarias-Universidad Nacional Autónoma de México, pp. 39-68.

Himanen, Pekka (2002). La ética del hacker y el espíritu de la era de la información. S.p.i. Disponible en http://eprints. rclis.org/1285l/

Levy, Steven (1984). Hackers: Heroes of the Computer Revolution. Garden City, Nueva York: Anchor Press, Doubleday.

Martín, Mario Toboso (2013). "Entre el uso y el no uso de la tecnología: un enfoque discursivo de la apropiación tecnológica". En Intersticios. Revista Sociológica de Pensamiento Crítico, 7(2), 201-214.

McKenzie, Wark (2004). A Hacker Manifesto. Harvard: President and Fellows of Harvard College.

Natansohn, Graciela (coord.) (2013). Internet en código femenino. Teorías y prácticas. Buenos Aires: La Crujía Ediciones.

Piñeiro, Erik (2003). The Aesthetics of Code: On Excellence in Instrumental Action. Estocolmo: Royal Institute of Technology.

Rivera Cusicanqui, Silvia (2018). Un mundo ch'ixi es posible. Ensayos desde un presente en crisis. Buenos Aires: Tinta Limón.
Rovira, Guiomar (2017). Activismo en red y multitudes conectadas. Comunicación y acción en la era de internet. Barcelona: Icaria.

Stallman, Richard M. (2004). Software libre para una sociedad libre. Madrid: Traficantes de Sueños.

Stiegler, Bernard (2002). La técnica y el tiempo. Hondarribia: Hiru.

Tabet, Paola (2005). "Las manos, los instrumentos, las armas". En Ochy Curiel y Jules Falquet (comps.), El patriarcado desnudo. Tres feministas materialistas. Buenos Aires: Brecha Lésbica.

Terrell, Josh, Andrew Kofink, Justin Middleton, Clarissa Rainear, Emerson Murphy-Hill, Chris Parnin y Jon Stallings (2017). "Gender differences and bias in open source: Pull request acceptance of women versus men". En PeerJ Computer Science, 3.

The Jargon File (2003). "The Jargon File (version 4.4.7)". Disponible en http://www.catb.org/-esr/jargon/html/ index.html

UNESCO (2019). Descifrar el código: Laeducaciónde las niñasy las mujeres en ciencias, tecnología, ingeniería y matemáticas (STEM). S.l:: Organización de las Naciones Unidas para la Educación, la Ciencia y la Cultura. Disponible en https:// es.unesco.org/themes/educacion-igualdad-genero/stem

Vergés Bosch, Nuria, Eva Cruells Lopez y Alex Haché (s/f). Descifrando el Código LeLa: Accesos, usos y deseos de las mujeres tecnólogas [documento inédito]. Disponible en https://www.academia.edu/1449913/Descifrando_ el_C\%C3\%B3digo_LeLa_Accesos_usos_y_deseos_de_la mujeres_tecn\%C3\%B3logas

Wajcman, Judith (2006). El tecnofeminismo. Valencia: Universitat de València.

Wiener, Norbert (1948). Cybernetics: Control and communication in the animal and the machine. Hoboken, Nueva Jersey: John Wiley \& Sons. 
Figura 1. Tipología empírica del uso de tecnología computacional de mujeres

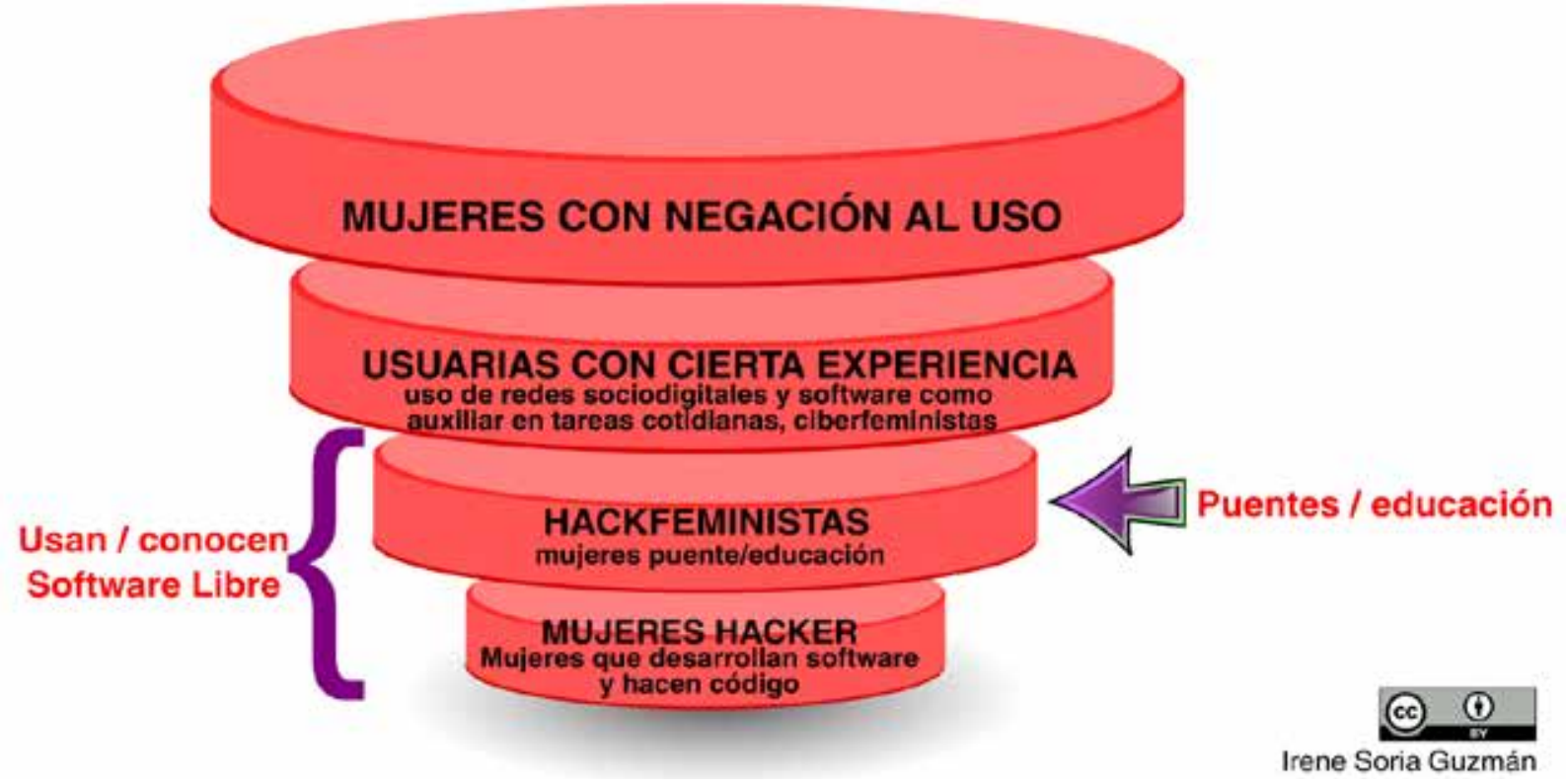

Fuente: elaboración propia.

Figura 2. Posición personal en la tipología empírica

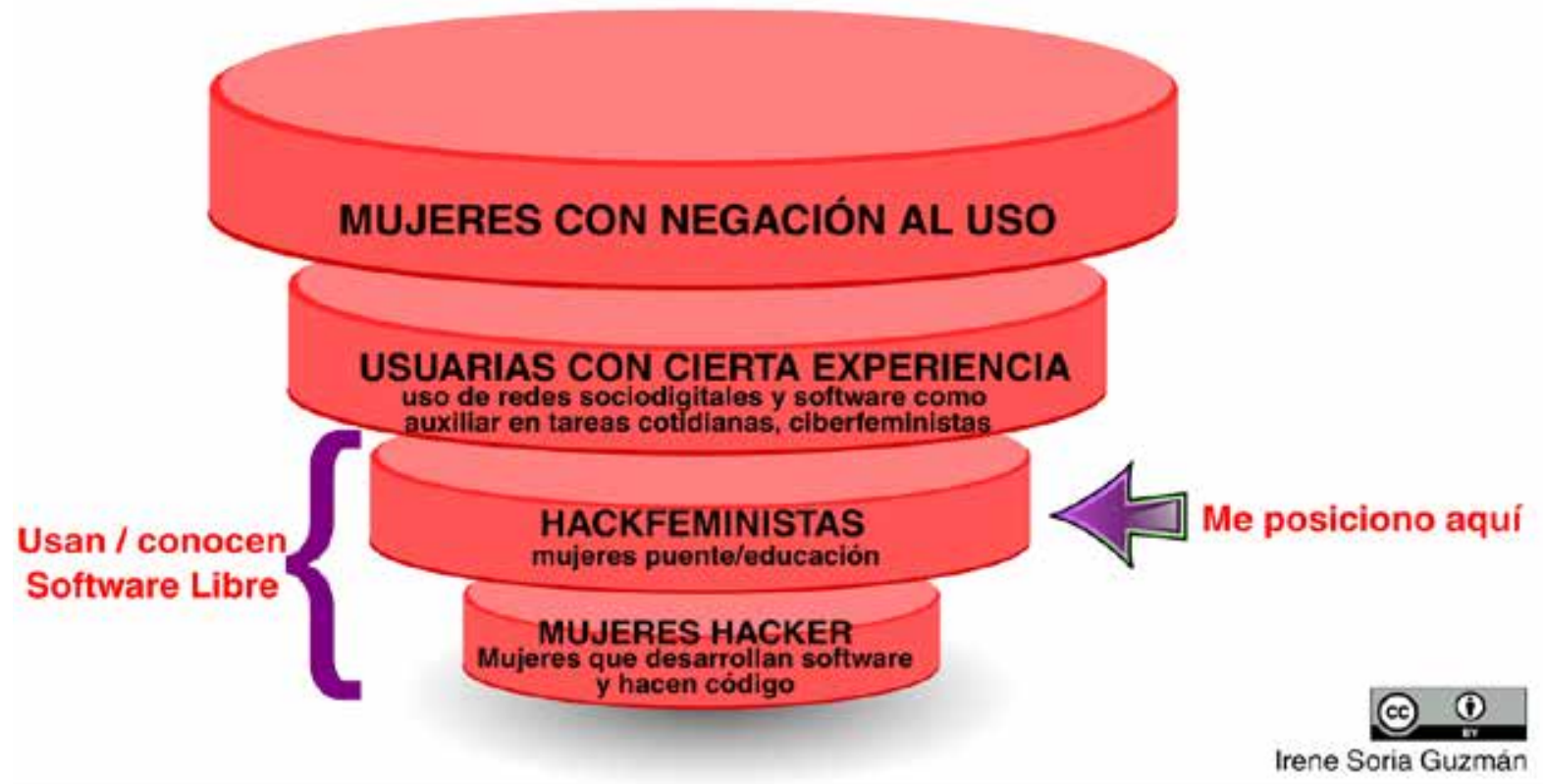

Fuente: elaboración propia. 
Figura 3. Proceso (personal) de apropiación tecnológica

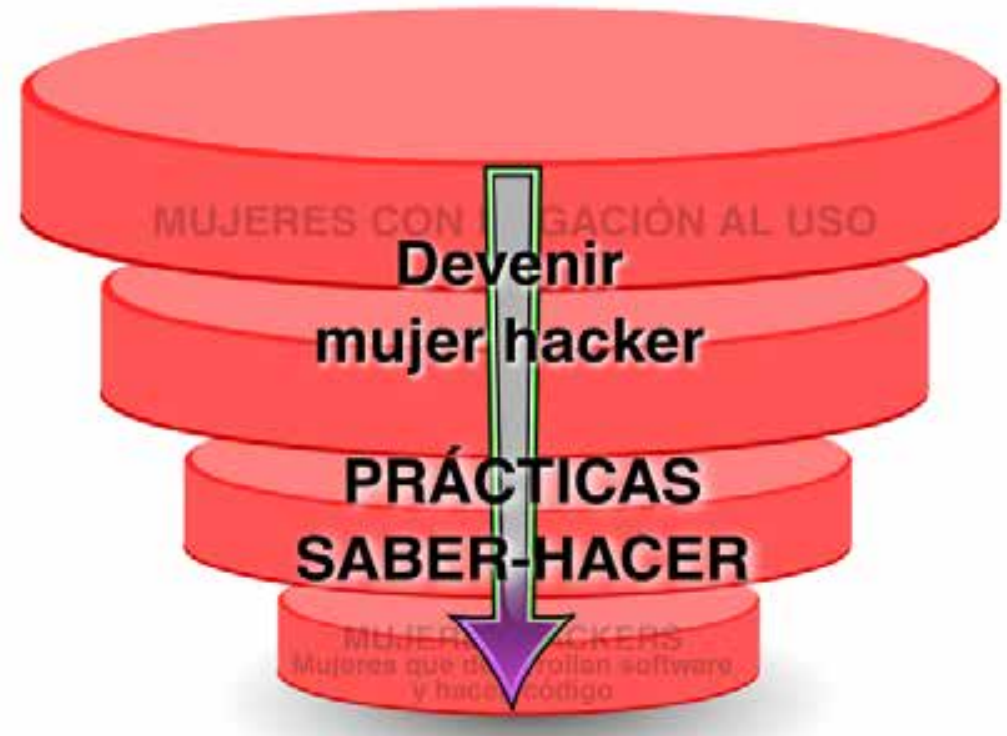

Irene Soria Guzmán

Fuente: elaboración propia.

\section{Cuadro 1. Perfiles de las mujeres entrevistadas}

\begin{tabular}{|c|c|c|c|}
\hline Edades & Regiones & Género & Clase \\
\hline $30-36: 6$ & Europa: 3 , & Mujer: 7 & Media: 6 \\
\hline $40,50: 2$ & Latinoamérica: 5 & No binaria: 1 & Trabajadora: 2 \\
\hline Raza/etnia & Estudios & Profesión & \\
\hline $\begin{array}{l}\text { Blancas: } 6 \\
\text { Mestizas: } 2\end{array}$ & $\begin{array}{l}\text { Doctorado: } 1 \\
\text { Universidad: } 5 \\
\text { Bachillerato: } 2\end{array}$ & $\begin{array}{l}\text { Sys-Admin: } 2 \\
\text { Desarrolladora/ } \\
\text { programadora: } 2 \\
\text { Líder de proyecto: } 2 \\
\text { Desarrollo Web: } 1 \\
\text { Manejo de hardware: } 1\end{array}$ & \\
\hline
\end{tabular}

Fuente: elaboración propia. 